International Journal of Pure and Applied Mathematics

Volume 87 No. 4 2013, 603-610

ISSN: 1311-8080 (printed version); ISSN: 1314-3395 (on-line version)

url: http://www.ijpam.eu

doi: http://dx.doi.org/10.12732/ijpam.v87i4.9

ijpam.eu

\title{
DIFFERENTIAL EQUATION OF GENERALIZED $K$-WRIGHT FUNCTION
}

\author{
Kuldeep Singh Gehlot ${ }^{1}$, Jyotindra C. Prajapati ${ }^{2}$, Bhailal P. Patel ${ }^{3}$ \\ ${ }^{1}$ Department of Mathematics \\ Government Bangur \\ P.G. College, Pali-306401, Rajasthan, INDIA \\ ${ }^{2}$ Department of Mathematical Sciences \\ Charotar University of Science and Technology \\ Changa, Anand, 388421, Gujarat, INDIA \\ ${ }^{3}$ Department of Mathematical Sciences \\ N.V. Patel College of Pure and Applied Sciences \\ Vallabh Vidyanagar, Gujarat, INDIA
}

\begin{abstract}
In this paper, authors introduced a homogeneous liner differential equation whose one of the solution in the form of Generalized K -Wright function. Special cases also deduced in terms of Generalized Wright function and Generalized $K$-Mittag-Leffler function.
\end{abstract}

AMS Subject Classification: 33B15, 33C20, 33E12, 34B30

Key Words: generalized $K$-wright function, $K$-Gamma function, $K$-Pochhammer symbol, generalized wright function, generalized $K$-Mittag-Leffler function

\section{Introduction}

Generalized $K$-Gamma Function $\Gamma_{k}(x)$ defined as (see Diaz and Pariguan [1])

Received: June 25, 2013

(C) 2013 Academic Publications, Ltd.

${ }^{\S}$ Correspondence author url: www.acadpubl.eu 


$$
\Gamma_{k}(x)=\lim _{n \rightarrow \infty} \frac{n ! k^{n}(n k)^{\frac{x}{k}-1}}{(x)_{n, k}}, k>0, x \in \mathbb{C} \backslash k \mathbb{Z}^{-},
$$

where $(x)_{n, k}$ is the $\mathrm{k}$-Pochhammer symbol and is given by

$$
(x)_{n, k}=x(x+k)(x+2 k) \ldots(x+(n-1) k), \quad x \in \mathbb{C}, k \in \mathbb{R}, n \in \mathbb{N}^{+}
$$

For $R e(x)>0, \Gamma_{k}(x)$ is $K$-Gamma function defined as the integral

$$
\Gamma_{k}(x)=\int_{0}^{\infty} t^{x-1} e^{\frac{-t^{k}}{k}} d t
$$

It is easy to prove following results

$$
\Gamma_{k}(x)=k^{\frac{x}{k}-1} \Gamma\left(\frac{x}{k}\right)
$$

and

$$
(\gamma)_{n q, k}=(k)^{n q}\left(\frac{\gamma}{k}\right)_{n q}
$$

The Generalized $K$-Wright function, introduced by [2], as

Definition 1. Generalized $K$-Wright Function is defined as ${ }_{p} \Psi_{q}^{k}(z)$ for $k \in$ $\mathbb{R}^{+} ; z \in \mathbb{C}, \alpha_{i}, \beta_{j} \in \mathbb{R}\left(\alpha_{i}, \beta_{j} \neq 0 ; i=1,2, \ldots, p ; j=1,2, \ldots, q\right)$ and $\left(a_{i}+\alpha_{i} n\right)$, $\left(b_{j}+\beta_{j} n\right) \in \mathbb{C} \backslash k \mathbb{Z}^{-}$,

$$
{ }_{p} \Psi_{q}^{k}(z)={ }_{p} \Psi_{q}^{k}\left[\begin{array}{c}
\left(a_{i}, \alpha_{i}\right)_{1, p} \\
\left(b_{j}, \beta_{j}\right)_{1, q}
\end{array} \mid z\right]=\sum_{n=0}^{\infty} \frac{\prod_{i=1}^{p} \Gamma_{k}\left(a_{i}+\alpha_{i} n\right) z^{n}}{\prod_{j=1}^{q} \Gamma_{k}\left(b_{j}+\beta_{j} n\right) n !}
$$

Theorem 1.1. (see Diaz and Pariguan [1]) For $k \in \mathbb{R}^{+} ; z \in \mathbb{C}, \alpha_{i}, \beta_{j} \in$ $\mathbb{R}\left(\alpha_{i}, \beta_{j} \neq 0 ; i=1,2, \ldots, p ; j=1,2, \ldots, q\right)$ and $\left(a_{i}+\alpha_{i} n\right),\left(b_{j}+\beta_{j} n\right) \in \mathbb{C} \backslash k \mathbb{Z}^{-}$,

(a) if $\Delta>-1$ then series (6) is absolutely convergent for all $z \in \mathbb{C}$ and Generalized $K$-Wright function ${ }_{p} \Psi_{q}^{k}(z)$ is an entire function of $z$.

(b) if $\Delta=-1$ then series (6) is absolutely convergent for all $|z|<\delta$ and of $|z|=\delta, \operatorname{Re}(\mu)>\frac{1}{2}$. 
We use following notations for describing convergence condition

$$
\begin{gathered}
\Delta=\sum_{j=1}^{q}\left(\frac{\beta_{j}}{k}\right)-\sum_{i=1}^{p}\left(\frac{\alpha_{i}}{k}\right) ; \delta=\prod_{i=1}^{p}\left|\frac{\alpha_{i}}{k}\right|^{\frac{-\alpha_{i}}{k}} \prod_{j=1}^{q}\left|\frac{\beta_{j}}{k}\right|^{\frac{\beta_{j}}{k}}, \\
\mu=\sum_{j=1}^{q}\left(\frac{b_{j}}{k}\right)-\sum_{i=1}^{p}\left(\frac{a_{i}}{k}\right)+\frac{p-q}{2} .
\end{gathered}
$$

The generalized Pochhammer symbol(Rainville [5]),

$$
(\gamma)_{n q}=\frac{\Gamma(\gamma+n q)}{\Gamma(\gamma)}=q^{q n} \prod_{r=1}^{q}\left(\frac{\gamma+r-1}{q}\right)_{n}, \quad \text { if } q \in \mathbb{N} .
$$

Equation (6) reduced in the following form by substituting $\alpha_{i}=k m_{i}, \beta_{j}=k l_{j}$; $m_{i}, l_{j} \in \mathbb{N}$ and using (4), (5), (7) we obtain,

$$
{ }_{p} \Psi_{q}^{k}(z)=A \sum_{n=0}^{\infty} \frac{\prod_{i=1}^{p} \prod_{r=1}^{m_{i}}\left(\frac{\frac{a_{i}}{k}+r-1}{m_{i}}\right)_{n}}{\prod_{j=1}^{q} \prod_{s=1}^{l_{j}}\left(\frac{\frac{b_{j}}{k}+s-1}{l_{j}}\right)_{n}} \frac{(B z)^{n}}{(n !)}
$$

where

$$
A=\frac{\prod_{i=1}^{p} \Gamma\left(\frac{a_{i}}{k}\right)}{\prod_{j=1}^{q} \Gamma\left(\frac{b_{j}}{k}\right)} K^{\left(\sum_{i=1}^{p} \frac{a_{i}}{k}-\sum_{j=1}^{q} \frac{b_{j}}{k}+q-p\right)}
$$

and

$$
B=\frac{\prod_{i=1}^{p}\left(m_{i}\right)^{m_{i}}}{\prod_{j=1}^{q}\left(l_{j}\right)^{l_{j}}} K^{\left(\sum_{i=1}^{p} m_{i}-\sum_{j=1}^{q} l_{j}\right)}
$$

\section{Main Result}

In this section, authors established linear homogeneous differential equation known as Generalized $K$-Wright differential equation. One of its solution is Generalized $K$-Wright function (8). Finally, this differential equation deduced in terms of Generalized Wright function and Generalized $K$-Mitteg-Leffler function.

Theorem 2.1. The Generalized K-Wright differential equation is defined as,

$$
\left[\theta \prod_{j=1}^{q} \prod_{s=1}^{l_{j}}\left(\theta+\frac{\frac{b_{j}}{k}+s-1}{l_{j}}-1\right)-B z \prod_{i=1}^{p} \prod_{r=1}^{m_{i}}\left(\theta+\frac{\frac{a_{i}}{k}+r-1}{m_{i}}\right)\right\rfloor W=0
$$


for

$$
p \leq q+1 \quad(i=1,2, \ldots, p \text { and } j=1,2, \ldots, q)
$$

where no $\frac{\frac{b_{j}}{k}+s-1}{l_{j}}$ is a negative integer or Zero and no two $\frac{\frac{b_{j}}{k}+s-1}{l_{j}}$ 's differ by an integer or Zero, then the solution is

$$
W=\sum_{t=0}^{q} C_{t} W_{t}
$$

where $C_{t}$ are arbitrary constant, and

$$
W_{0}={ }_{p} \Psi_{q}^{k}(z)
$$

and for $t=1,2,3, \ldots, q$

$$
\begin{aligned}
& W_{t}= \\
& \quad \sum_{n=0}^{\infty} \frac{\prod_{i=1}^{p} \prod_{r=1}^{m_{i}}\left(1+\frac{\frac{a_{i}}{k}+r-1}{m_{i}}-\prod_{s=1}^{l_{t}} \frac{\frac{b_{t}}{k}+s-1}{l_{t}}\right)_{n}}{\prod_{j=1}^{q} \prod_{s=1}^{l_{j}}\left(1+\frac{\frac{b_{j}}{k}+s-1}{l_{t}}-\prod_{s=1}^{l_{t}} \frac{\frac{b_{t}}{k}+s-1}{l_{t}}\right)_{n}} \frac{(B z)^{n+1-\prod_{s=1}^{l_{t}}\left(\frac{\frac{b_{t}}{k}+s-1}{l_{t}}\right)}}{\left(2-\prod_{s=1}^{l_{t}} \frac{\frac{b_{t}}{k}+s-1}{l_{t}}\right)_{n}} .
\end{aligned}
$$

Here $\theta=z \frac{d}{d z}$ and $B$ is given by (10).

Proof. From given condition, no $\frac{\frac{b_{j}}{k}+s-1}{l_{j}}$ is a positive integer then the linear combination (12) is the general solution of equation (11) around $z=0$. Note that if $p \leq q(i=1,2, \ldots, p$ and $j=1,2, \ldots, q)$ then the series $W_{t} ; t=$ $0,1,2, \ldots, q$, converge for all finite $\mathrm{z}$ and for $p \leq q+1,(i=1,2, \ldots, p$ and $j=$ $1,2, \ldots, q)$ the series $W_{t}$ converges for $|z|<1$.

To verify that $W_{0}$, satisfies (11).

Consider,

$$
\Delta \equiv \theta \prod_{j=1}^{q} \prod_{s=1}^{l_{j}}\left(\theta+\frac{\frac{b_{j}}{k}+s-1}{l_{j}}-1\right) W_{0}
$$

using (8), it follows that

$$
\Delta \equiv A \sum_{n=0}^{\infty} \frac{\theta \prod_{j=1}^{q} \prod_{s=1}^{l_{j}}\left(\theta+\frac{\frac{b_{j}}{k}+s-1}{l_{j}}-1\right) \prod_{i=1}^{p} \prod_{r=1}^{m_{i}}\left(\frac{\frac{a_{i}}{k}+r-1}{m_{i}}\right)_{n}}{\prod_{j=1}^{q} \prod_{s=1}^{l_{j}}\left(\frac{\frac{b_{j}}{k}+s-1}{l_{j}}\right)_{n}} \frac{(B z)^{n}}{(n !)}
$$


where A and B are given by (9) and (10), respectively.

Since $\theta(B z)^{n}=n(B z)^{n}$, we have

$$
\Delta \equiv A \sum_{n=0}^{\infty} \frac{n \prod_{j=1}^{q} \prod_{s=1}^{l_{j}}\left(n+\frac{\frac{b_{j}}{k}+s-1}{l_{j}}-1\right) \prod_{i=1}^{p} \prod_{r=1}^{m_{i}}\left(\frac{\frac{a_{i}}{k}+r-1}{m_{i}}\right)_{n}}{\prod_{j=1}^{q} \prod_{s=1}^{l_{j}}\left(\frac{\frac{b_{j}}{k}+s-1}{l_{j}}\right)_{n}} \frac{(B z)^{n}}{(n !)},
$$

this gives,

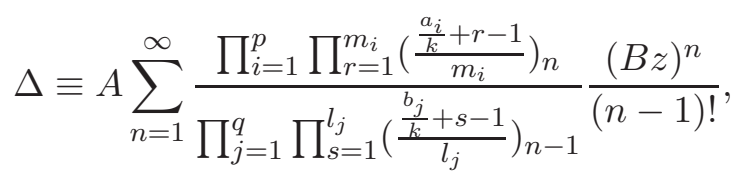

above equation can be written as,

$$
\Delta \equiv A \sum_{n=0}^{\infty} \frac{\prod_{i=1}^{p} \prod_{r=1}^{m_{i}}\left(\frac{\frac{a_{i}}{k}+r-1}{m_{i}}\right)_{n+1}}{\prod_{j=1}^{q} \prod_{s=1}^{l_{j}}\left(\frac{\frac{b_{j}}{k}+s-1}{l_{j}}\right)_{n}} \frac{(B z)^{n+1}}{(n) !}
$$

above equation reduces to,

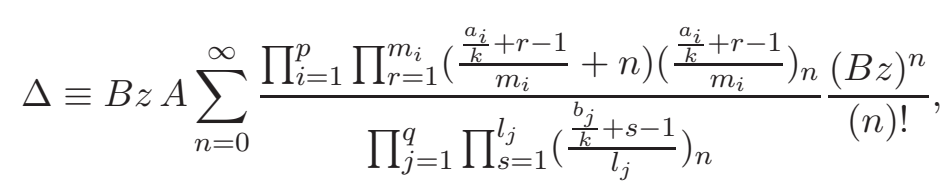

this leads,

$$
\Delta \equiv B z \prod_{i=1}^{p} \prod_{r=1}^{m_{i}}\left(\theta+\frac{\frac{a_{i}}{k}+r-1}{m_{i}}\right) W_{0} .
$$

This shows that $W_{0}$ is a solution of the differential equation (11).

To verify that $W_{t}, t=1,2, \ldots, q$, satisfies equation (11).

Consider,

$$
\Omega \equiv \theta \prod_{j=1}^{q} \prod_{s=1}^{l_{j}}\left(\theta+\frac{\frac{b_{j}}{k}+s-1}{l_{j}}-1\right) W_{t}
$$

from (14), we get

$$
\Omega \equiv \theta \prod_{j=1}^{q} \prod_{s=1}^{l_{j}}\left(\theta+\frac{\frac{b_{j}}{k}+s-1}{l_{j}}-1\right) \sum_{n=0}^{\infty} \frac{\prod_{i=1}^{p} \prod_{r=1}^{m_{i}}\left(1+\frac{\frac{a_{i}}{k}+r-1}{m_{i}}-\prod_{s=1}^{l_{t}} \frac{\frac{b_{t}}{k}+s-1}{l_{t}}\right)_{n}}{\prod_{j=1}^{q} \prod_{s=1}^{l_{j}}\left(1+\frac{\frac{b_{j}}{k}+s-1}{l_{t}}-\prod_{s=1}^{l_{t}} \frac{\frac{b_{t}}{k}+s-1}{l_{t}}\right)_{n}}
$$




$$
\times \frac{(B z)^{n+1-\prod_{s=1}^{l_{t}}\left(\frac{\frac{b_{t}}{k}+s-1}{l_{t}}\right)}}{\left(2-\prod_{s=1}^{l_{t}} \frac{\frac{b_{t}}{k}+s-1}{l_{t}}\right)_{n}}
$$

Since $\theta(B z)^{n}=n(B z)^{n}$, we have

$$
\begin{aligned}
\Omega \equiv & \sum_{n=0}^{\infty} \frac{\left(n+1-\prod_{s=1}^{l_{t}}\left(\frac{\frac{b_{t}}{k}+s-1}{l_{t}}\right)\right) \prod_{j=1}^{q} \prod_{s=1}^{l_{j}}\left(n-\prod_{s=1}^{l_{t}}\left(\frac{\frac{b_{t}}{k}+s-1}{l_{t}}\right)+\frac{\frac{b_{j}}{k}+s-1}{l_{j}}\right)}{\prod_{j=1}^{q} \prod_{s=1}^{l_{j}}\left(1+\frac{\frac{b_{j}}{k}+s-1}{l_{t}}-\prod_{s=1}^{l_{t}} \frac{\frac{b_{t}}{k}+s-1}{l_{t}}\right)_{n}} \\
& \times \frac{\prod_{i=1}^{p} \prod_{r=1}^{m_{i}}\left(1+\frac{\frac{a_{i}}{k}+r-1}{m_{i}}-\prod_{s=1}^{l_{t}} \frac{\frac{b_{t}}{k}+s-1}{l_{t}}\right)_{n}(B z)^{n+1-\prod_{s=1}^{l_{t}}\left(\frac{b_{t}+s-1}{l_{t}}\right)}}{\left(2-\prod_{s=1}^{l_{t}} \frac{\frac{b_{t}}{k}+s-1}{l_{t}}\right)_{n}}
\end{aligned}
$$

On simplification, we have,

$$
\frac{\left(n+1-\prod_{s=1}^{l_{t}}\left(\frac{\frac{b_{t}}{k}+s-1}{l_{t}}\right)\right)}{\left(2-\prod_{s=1}^{l_{t}} \frac{\frac{b_{t}}{k}+s-1}{l_{t}}\right)_{n}}=\frac{1}{\left(2-\prod_{s=1}^{l_{t}} \frac{\frac{b_{t}}{k}+s-1}{l_{t}}\right)_{n-1}} .
$$

and

$$
\begin{gathered}
\frac{\prod_{j=1}^{q} \prod_{s=1}^{l_{j}}\left(n-\prod_{s=1}^{l_{t}}\left(\frac{\frac{b_{t}}{k}+s-1}{l_{t}}\right)+\frac{\frac{b_{j}}{k}+s-1}{l_{j}}\right)}{\prod_{j=1}^{q} \prod_{s=1}^{l_{j}}\left(1+\frac{\frac{b_{j}}{k}+s-1}{l_{t}}-\prod_{s=1}^{l_{t}} \frac{\frac{b_{t}}{k}+s-1}{l_{t}}\right)_{n}} \\
=\frac{1}{\prod_{j=1}^{q} \prod_{s=1}^{l_{j}}\left(1+\frac{\frac{b_{j}}{k}+s-1}{l_{t}}-\prod_{s=1}^{l_{t}} \frac{\frac{b_{t}}{k}+s-1}{l_{t}}\right)_{n-1}}
\end{gathered}
$$

substituting (16) and (17) in (15), we obtain

$$
\Omega \equiv \sum_{n=1}^{\infty} \frac{\prod_{i=1}^{p} \prod_{r=1}^{m_{i}}\left(1+\frac{\frac{a_{i}}{k}+r-1}{m_{i}}-\prod_{s=1}^{l_{t}} \frac{\frac{b_{t}}{k}+s-1}{l_{t}}\right)_{n}}{\prod_{j=1}^{q} \prod_{s=1}^{l_{j}}\left(1+\frac{\frac{b_{j}}{k}+s-1}{l_{t}}-\prod_{s=1}^{l_{t}} \frac{\frac{b_{t}}{k}+s-1}{l_{t}}\right)_{n-1}} \frac{\left(2-\prod_{s=1}^{l_{t}} \frac{\frac{b_{t}}{k}+s-1}{l_{t}}\right)_{n-1}}{\left(2+1-\prod_{s=1}^{l_{t}}\left(\frac{\frac{b_{t}}{k}+s-1}{l_{t}}\right)\right.},
$$

now we replace $n$ by $n+1$, we get

$$
\Omega \equiv B z \sum_{n=0}^{\infty} \frac{\prod_{i=1}^{p} \prod_{r=1}^{m_{i}}\left(1+\frac{\frac{a_{i}}{k}+r-1}{m_{i}}-\prod_{s=1}^{l_{t}} \frac{\frac{b_{t}}{k}+s-1}{l_{t}}\right)_{n+1}}{\prod_{j=1}^{q} \prod_{s=1}^{l_{j}}\left(1+\frac{\frac{b_{j}}{k}+s-1}{l_{t}}-\prod_{s=1}^{l_{t}} \frac{\frac{b_{t}}{k}+s-1}{l_{t}}\right)_{n}} \frac{\left.(B z)^{n+1-\prod_{s=1}^{l_{t}}\left(\frac{b_{t}}{k}+s-1\right.} l_{t}\right)}{\left(2-\prod_{s=1}^{l_{t}} \frac{\frac{b_{t}}{k}+s-1}{l_{t}}\right)_{n}} .
$$


From the equation above, it follows that

$$
\begin{aligned}
& \Omega \equiv \\
& B z \sum_{n=0}^{\infty} \frac{\prod_{i=1}^{p} \prod_{r=1}^{m_{i}}\left(n+1+\frac{\frac{a_{i}}{k}+r-1}{m_{i}}-\prod_{s=1}^{l_{t}} \frac{\frac{b_{t}}{k}+s-1}{l_{t}}\right)\left(1+\frac{\frac{a_{i}}{k}+r-1}{m_{i}}-\prod_{s=1}^{l_{t}} \frac{\frac{b_{t}}{k}+s-1}{l_{t}}\right)_{n}}{\prod_{j=1}^{q} \prod_{s=1}^{l_{j}}\left(1+\frac{\frac{b_{j}}{k}+s-1}{l_{t}}-\prod_{s=1}^{l_{t}} \frac{\frac{b_{t}}{k}+s-1}{l_{t}}\right)_{n}} \\
& \times \frac{(B z)^{n+1-\prod_{s=1}^{l_{t}}\left(\frac{\frac{b_{t}}{k}+s-1}{l_{t}}\right)}}{\left(2-\prod_{s=1}^{l_{t}} \frac{\frac{b_{t}}{k}+s-1}{l_{t}}\right)_{n}},
\end{aligned}
$$

Hence,

$$
\left.\Omega \equiv B z \prod_{i=1}^{p} \prod_{r=1}^{m_{i}}\left(\theta+\frac{\frac{a_{i}}{k}+r-1}{m_{i}}\right)\right] W_{t} .
$$

This shows that $W_{t}, t=1,2, \ldots, q$ is the solutions of the differential equation (11).

Particular Cases: For suitable values of the parameters, we can obtain certain differential equations for different generalized functions for $\alpha_{i}=k m_{i}$, $\beta_{j}=k l_{j} ; m_{i}, l_{j} \in \mathbb{N} ; i=1,2, \ldots, p ; j=1,2, \ldots, q$ and $k \in \mathbb{R}$.

[A] Taking $k=1$ in (11), we have arrived at

$$
\left[\theta \prod_{j=1}^{q} \prod_{s=1}^{l_{j}}\left(\theta+\frac{b_{j}+s-1}{l_{j}}-1\right)-B z \prod_{i=1}^{p} \prod_{r=1}^{m_{i}}\left(\theta+\frac{a_{i}+r-1}{m_{i}}\right) \mid W=0 .\right.
$$

where $B=\frac{\prod_{i=1}^{p}\left(m_{i}\right)^{m_{i}}}{\prod_{j=1}^{q}\left(l_{j}\right)^{l_{j}}}$.

Equation (18), is the differential equation for Generalized Wright function $W_{0}={ }_{p} \Psi_{p}(z)$, definded by Wright [10].

[B] Taking $p=1, q=2 ; \alpha_{1}=k m_{1}, \beta_{1}=k l_{1}, \beta_{2}=0$ in (11), we obtain,

$$
\left[\theta \prod_{j=1}^{2} \prod_{s=1}^{l_{j}}\left(\theta+\frac{b_{j}+s-1}{l_{j}}-1\right)-B z \prod_{i=1}^{1} \prod_{r=1}^{m_{i}}\left(\theta+\frac{a_{i}+r-1}{m_{i}}\right)\right\rfloor W=0
$$

where

$$
B=\frac{\prod_{i=1}^{1}\left(m_{i}\right)^{m_{i}}}{\prod_{j=1}^{2}\left(l_{j}\right)^{l_{j}}} K^{\left(\sum_{i=1}^{1} m_{i}-\sum_{j=1}^{2} l_{j}\right)}
$$


Equation (19), is the differential equation for Generalized $K$-Mittag- Leffler function $G E_{k, k m_{1}, k l_{1}}^{a_{1}, m_{1}}(z)$, definded by Gehlot [3,4].

The function defined in equation (10) is an extension of generalized MittagLeffler function defined by Shukla and Prajapati ([6], [7], [8]), Shukla et al [9].

\section{References}

[1] R. Diaz, E. Pariguan, On hypergeometric functions and Pochhammer $k$ symbol, Divulgaciones Mathematicas, 15 No. 2 (2007), 179-192.

[2] Gehlot Kuldeep Singh, Jyotindra C. Prajapati, Fractional calculus of generalized $K$-wright function, Journal of Fractional Calculus and Applications, 4, No. 2 (2013), 283-289.

[3] Kuldeep Singh Gehlot, Differential equation of generalized $K$-MittagLeffler function, Int. J. Contemp. Math. Sciences (2013), Accepted.

[4] Kuldeep Singh Gehlot, The generalized K-Mittag-Leffler function, Int. J. Contemp. Math. Sciences, 7, No. 45 (2012), 2213-2219.

[5] Earl D. Rainville, Special Functions, The Macmillan Company, New York (1963).

[6] A.K. Shukla, J.C. Prajapati, A general class of polynomials associated with generalized Mittag-Lefflerfunction, Integral Transforms and Special Functions, 19, No. 1 (2008), 23-34.

[7] A.K. Shukla, J.C. Prajapati, Some remarks on generalized Mittag-Leffler function, Proyecciones Journal of Mathematics, 28, No. 1 (2009), 27-34.

[8] A.K. Shukla, J.C. Prajapati, On a generalized Mittag-Leffler type function and generated integral operator, Mathematical Sciences Research Journal, 12 (2008), 283-290.

[9] A.K. Shukla, I.A. Salehbhai, J.C. Prajapati, On Laguerre transform in two variables, Integral Transforms and Special Functions, 20, No. 6 (2009), 459-470.

[10] E.M. Wright, The asymptotic expansion of the grneralized hypergeometric function, J. London Math. Soc., 10 (1935), 287-293. 